TAMKANG JOURNAL OF MATHEMATICS

Volume 33, Number 1, Spring 2002

\title{
SIMPLE RINGS OF CHARACTERISTIC NOT 2 WITH ASSOCIATORS IN THE LEFT NUCLEUS ARE ASSOCIATIVE
}

\author{
CHEN-TE YEN
}

\begin{abstract}
We prove that if $R$ is a simple ring of characteristic not 2 with associators in the left nucleus then $R$ is associative. This extends our previous result [2].
\end{abstract}

\section{Introduction}

Let $R$ be a nonassociative ring. We shall denote the associator by $(x, y, z)=(x y) z-$ $x(y z)$ for all $x, y, z$ in $R$. In any ring $R$ one has the following nuclei:

$$
\begin{aligned}
N & =\{n \in R \mid(n, R, R)=0\}-\text { left nucleus, } \\
M & =\{n \in R \mid(R, n, R)=0\}-\text { middle nucleus, } \\
L & =\{n \in R \mid(R, R, n)=0\}-\text { right nucleus. }
\end{aligned}
$$

A ring $R$ is called simple if $R^{2} \neq 0$ and the only nonzero ideal of $R$ is itself. Since $R^{2}$ is a nonzero ideal of $R$, we have $R^{2}=R$. A ring $R$ is called semiprime if the only ideal of $R$ which squares to zero is the zero ideal. Note that each associator is linear in each argument. Thus $N, M$ and $L$ are additive subgroups of $(R,+)$. We shall use the Teichmüller identity.

(1) $(w x, y, z)-(w, x y, z)+(w, x, y z)=w(x, y, z)+(w, x, y) z$ for all $w, x, y, z$ in $R$, which is valid in every ring.

As a consequence of (1), we have that $N, M$ and $L$ are associative subrings of $R$. Suppose that $n \in N$. Then with $w=n$ in (1) we obtain

(2) $(n x, y, z)=n(x, y, z)$ for all $x, y, z$ in $R$ and $n$ in $N$.

Definition. Let $A$ be the associator ideal of a ring $R$.

By (1) $A$ can be characterized as all finite sums of associators and right (or left) multiples of associators. Hence, we otbain

(3) $A=(R, R, R)+(R, R, R) R$.

Received September 22, 1999; revised September 21, 2001.

2000 Mathematics Subject Classification. Primary 17A30.

Key words and phrases. Nucleus, simple ring, semiprime ring, associator ideal. 
In [1], E. Kleinfeld proved that if $R$ is a semiprime ring such that $(R, R, R) \subseteq N \cap M \cap L$ and $(R,+)$ has no elements of order 2 then $R$ is associative. In [2], the author showed the result for the simple ring case under the weaker hypothesis $(R, R, R) \subseteq N \cap M$. In the note, we extends this result. In [3], we generalized E. Kleinfeld's result under the weaker hypothesis $(R, R, R) \subseteq$ two of the three nuclei.

\section{Result}

Theorem. Let $R$ be a simple ring of characteristic not 2 and satisfy $(*)(R, R, R) \subseteq$ $N$. Then $R$ is associative.

Proof. Assume that $R$ is not associative. Then by $(3)$ and $(*)$, we have

(4) $R=R^{2}=A R=\{(R, R, R)+(R, R, R) R\} R=(R, R, R) R+(R, R, R) R^{2}=(R, R, R) R$.

Using (1) and (*), we get

(5) $w(x, y, z)+(w, x, y) z \in N$ for all $w, x, y, z$ in $R$.

Then with $x \in(R, R, R)$ in (5), and applying (*) we obtain $(R,(R, R, R), R) R \subseteq N$. Using this, $(*)$ and $(2)$, we have $0=((R,(R, R, R), R) R, R, R)=(R,(R, R, R), R)(R, R, R)$ and so $(R,(R, R, R), R) \cdot(R, R, R) R=(R,(R, R, R), R)(R, R, R) \cdot R=0$. Combined this with (4) results in

(6) $(R,(R, R, R), R) R=0$.

Assume that $x \in(R,(R, R, R), R)$ and $w, y, z, t \in R$. Then by (6), (1) and (*) we get $(w x, y, z)+(w, x, y z)=(w x, y, z)-(w, x y, z)+(w, x, y z)=w(x, y, z)+(w, x, y) z=0$ and so $(w x, y, z) t=-(w, x, y z) t=0$. The last identity implies

(7) $(R(R,(R, R, R), R), R, R) R=0$.

Then with $y \in(R, R, R)$ in (5) and applying (7), we obtain

(8) $((R, R,(R, R, R)) R, R, R) R=0$.

Using (4), (*), (2) and (8), we have

(9) $(R, R,(R, R, R)) R=(R, R,(R, R, R)) \cdot(R, R, R) R=(R, R,(R, R, R))(R, R, R) \cdot R=$ $((R, R,(R, R, R)) R, R, R) R=0$.

Then with $y \in(R, R, R)$ in (5) and applying (9), we get

(10) $R(R,(R, R, R), R) \subset N$.

For all $x \in(R,(R, R, R), R)$ and $w, y, z \in R$, using the previous computation and by (10) we obtain $(w, x, y z)=-(w x, y, z)=0$.

Since $R^{2}=R$, this implies 
(11) $(R,(R, R, R), R) \subseteq M$.

Let $T=(R,(R, R, R), R)$. We define $V_{n}$ inductively by $V_{0}=T, V_{1}=R T$ and $V_{n+1}=R V_{n}, n=1,2,3, \cdots$. Assume that

(12) $B=\sum_{n=0}^{\infty} V_{n}$.

We want to prove by induction that

(13) $B \cdot R=0$

By (6), (11) and (9), we have $V_{0} R=T R=0, V_{1} R=R T \cdot R=R \cdot T R=0$ and $V_{2} R=$ $R(R T) \cdot R \subseteq\left((R, R, T)+R^{2} T\right) R=(R, R, T) R+R^{2} T \cdot R=0$. Suppose that $V_{i} R=0$, $i=0,1,2, \cdots, m$ and $V_{m+1} R=0$. Then using these and (9), we get $V_{m+2} R=R\left(R V_{m}\right)$. $R \subseteq\left(\left(R, R, V_{m}\right)+R^{2} V_{m}\right) R=\left(R, R, V_{m}\right) R+V_{m+1} R=\left(R, R, V_{m}\right) R=\left(R, R, R\left(R V_{m-2}\right)\right) R \subseteq$ $\left(R, R,\left(R, R, V_{m-2}\right)+R^{2} V_{m-2}\right) R=\left(R, R,\left(R, R, V_{m-2}\right)\right) R+\left(R, R, V_{m-1}\right) R=\left(R, R, V_{m-1}\right) R$. Continuing in this manner, we eventually have $V_{m+2} R \subseteq\left(R, R, V_{m}\right) R \subseteq\left(R, R, V_{m-1}\right) R$ $\subseteq \cdots \subseteq\left(R, R, V_{2}\right) R \subseteq\left(R, R, V_{1}\right) R=(R, R, R T) R$. By (1) and (9), we get $R T=$ $R(R,(R, R, R), R) \subseteq(R, R, R)+(R, R,(R, R, R)) R=(R, R, R)$.

Thus, applying this and (9) we have $V_{m+2} R \subseteq(R, R, R T) R \subseteq(R, R,(R, R, R)) R$ $=0$. Hence, by induction (13) holds. By (13), $B$ is just the ideal of $R$ generated by $(R,(R, R, R), R)$. By the simplicity of $R$ and (13), we get $B=0$. Thus, $(R,(R, R, R), R)$ $=0$ and so $(R, R, R) \subseteq N \cap M$. Hence, by Theorem 2 of [2], $R$ is associative. This contradiction proves the theorem.

\section{References}

[1] E. Kleinfeld, A class of rings which are very nearly associative, Amer. Math. Monthly, 93(1986), 720-722. MR. 87j:17003.

[2] C. T. Yen, Rings with associators in the left and middle nucleus, Tamkang J. Math., 23(1992), 363-369. MR.93k:17003.

[3] C. T. Yen, Rings with associators in the nuclei, Chung Yuan J., 28(2000), 7-9.

Department of Mathematics, Chung Yuan Christian University, Chung Li, Taiwan, 320, Republic of China. 